\title{
LEXIKALISCHER WANDEL AM BEISPIEL DES VERBS STILLEN
}

\section{EINLEITUNG}

Sprache, vor allem die Lexik, wandelt sich über längere Zeiträume hinweg. Sprachwissenschaftler beobachten diese Prozesse, beschreiben sie, diskutieren mögliche Ursachen und leiten Tendenzen ab. So hat das auch Prof. Siegfried Heusinger bis heute getan. Vielen Studentinnen und Studenten im In- und Ausland hat er die lebendige deutsche Sprache nahegebracht. Seine Auffassung zu Bedeutungswandel ist u. a. in der Einführung zur Lexik der deutschen Gegenwartssprache im Kapitel 5.2. nachzulesen (Heusinger 2004: 169ff.).

Am Beispiel des Verbs stillen soll exemplarisch solch ein lexikalischer Wandel dargestellt werden, der sich möglicherweise durch die Nutzung von neuen Kulturtechniken wie Internetplattformen in relativ kurzer Zeit vollzieht und sich entsprechend belegen lässt.

\section{DISKUSSION AUSGEWÄHLTER BEISPIELE}

Im Internet findet sich das Forum www.stillen-und-tragen.de, das Müttern seit einigen Jahren die Möglichkeit bietet, sich über sämtliche Aspekte des Mutterseins auszutauschen. Ein besonderer Schwerpunkt ist das Stillen von Babys. Dieses Forum ist inzwischen im gesamten deutschsprachigen Raum bekannt. Stillen, auch über den ersten Geburtstag des Kindes hinaus, so genanntes Langzeitstillen, ist in dem Forum ein weit verbreitetes Thema. Darüber hinaus bieten ausgebildete Stillberaterinnen hier eine Online-Stillberatung an. Hier einige Auszüge aus Beiträgen dieser Internetplattform, die als Belegmaterial für die anschließende Diskussion der Frage nach lexikalischem Wandel des Verbs dienen sollen:

[...] Unser 5-jähriges Nachbarskind fragt jetzt immer in einem vorwurfsvollen Ton „Warum stillt die Fiona noch? Das machen doch nur Babys!“ Er würde ja auch nicht mehr stillen. [...] (22.1.12); Gestern nachmittag wurde mein Sohn müde, wollte stillen und etwas dösen [...] (3.2.13); [... ... Mein SchwieMu hat Bärchen während er gestillt hat den Kopf gestreichelt. [...] (17.2.14); [...] Wenn er abgedockt hat frage ich ihn meistens ob's geschmeckt hat, da grinst er nur... ach ich stille einfach so gerne [...] (8.9.15); [...] Nur einmal, als ich absolut keine Lust mehr hatte, sie aber unbedingt wollte, hat sie auf einmal bitterlich „stiiiiiillen“ gerufen. Das war bisher das einzige Mal, dass sie das Wort stillen benutzt hat, [...]. (6.1.16);

christina.janz@tu-dresden.de 
[... ... mein Sohn macht Yoga - er stillt seit neuestem im Kopfstand (wir stillen meist im liegen) [...] (14.9.16); [...] L stillt gerne neben mir kniend und fängt dann an, den Po in die Luft zu strecken. [...] (23.2.17); [...] Wenn der kleine Mann im Halbschlaf stillt und versehentlich los lässt und [...] (2.4.18); [...] Heute morgen wollte der kleine Mann stillen, ich hab ihn auf die Tasse Kuhmilch verwiesen, die auf dem Tisch stand. Was macht er? Holt die Tasse, hält sie mir an den Mund und lässt mich trinken. Wiederholt das so lange bis die Tasse leer ist, sagt alle, stellt die Tasse weg und wirft sich in Stillposition [...] (16.12.18)

Dieser kurze Ausschnitt von Beispielen zeigt, dass mindestens seit 2012 das Verb stillen auf der genannten Internetplattform intransitiv verwendet wird, mit dem jeweiligen Kind als Subjekt. In wenigen Beispielen, in denen Kinder zu Wort kommen, die bereits drei Jahre und älter sind, wird das Verb von ihnen selbst intransitiv verwendet. Die intransitive Verwendung des Verbs durch verschiedene Erwachsene findet sich in dem Forum seit etwa 2015 recht häufig. Eine valide Analyse kann an dieser Stelle nicht geleistet werden. In Grammatiken wird zurzeit noch allein die transitive Verwendung des Verbs stillen als standardsprachlich üblich betrachtet.

\section{EXKURS ZUR ETYMOLOGIE DES VERBS STILLEN}

Etymologisch gesehen handelt es sich bei stillen um ein schwaches, transitives Verb.

Im Althochdeutschen bedeutet es 'stillen', 'beruhigen', 'zur Ruhe bringen', 'zum Schweigen bringen', 'heilen von', 'bezähmen', 'beruhigen` (Köbler 1994: 302). Im Mittelhochdeutschen Wörterbuch wird stillen wie folgt beschrieben: 1. transitiv: stille machen, zur Ruhe, zum Schweigen bringen, beruhigen, besänftigen, zurück-, aufhalten, hindern, abbringen von, befriedigen, geheim halten; 2 . intransitiv: still werden, zur Ruhe kommen, sich besänftigen, aufhören, schweigen, ablassen von (Lexer 1992: 211). Im Etymologischen Wörterbuch der deutschen Sprache heißt es dazu: [...] zum Schweigen bringen, säugen erw. fach. (8. Jh.), mhd. Stillen, ahd. Stillen, as. (g)stillan. [...] Die Bedeutung „einem Kind die Brust geben“ beruht auf der Bedeutungsverschiebung aus „es zum Schweigen bringen“, vielleicht als Hüllwort. (Kluge 2002: 885) Weiter wird ausgeführt, dass vor dem 16. Jahrhundert für stillen auch säugen üblich war (althochdeutsch: sugan, mittelhochdeutsch: sugen oder sougen). Der Gebrauch ist in dieser Zeit transitiv und intransitiv. Das Wort Säugling ist eine Nominalableitung von säugen. (Kluge 2002: 787) Im Kleinen Wahrig steht zum Verb stillen 1. Einen Säugling an der Mutterbrust trinken lassen, nähren, 2. Etwas zum Stillstand bringen; Blut, 3. Ein Bedürfnis befriedigen, seinen Durst, Hunger stillen (Wahrig 1982: 745).

Standardsprachlich ordnen die Grammatiken zur deutschen Gegenwartssprache das Verb stillen den transitiven Verben zu. Transitive Verben sind in diesem Verständnis solche, die ein Akkusativobjekt bei sich haben, das bei der sog. Passivtransformation zum Subjekt wird. Also: Die Mutter stillt das Kind. Das Kind wird gestillt. 
Das Akkusativobjekt ist nicht zwingend erforderlich, z. B. ich stille gern. Intransitive Verben hingegen sind Verben, die kein Akkusativobjekt bei sich haben und die außer dem Subjekt im Satz keine weiteren Argumente/Beteiligte zulassen. Transitive Verben mit zwei Beteiligten in der Satzbildung lassen prototypisch die Unterscheidung der syntaktisch-semantischen Rollen von Agens und Patiens zu. Viele Verben in der deutschen Gegenwartssprache werden sowohl transitiv als auch intransitiv verwendet. Ein Beispiel aus dem hier betrachteten Diskurs für diese auch ergativ genannten Verben: Das Baby badet einmal in der Woche. Ich bade das Baby.

In den Beispielen von der Internetplattform wird aber das Verb stillen intransitiv verwendet: Das Kind stillt. Evtl. kann von einer Analogiebildung zu das Kind trinkt ausgegangen werden. Damit scheint mit der intransitiven Verwendung des Verbs stillen etwas ausgedrückt zu werden, was das Kind aktiv tut, was aber eben nicht trinken oder essen ist.

\section{Warum gibt es die intransitive Nutzung dieses Verbs? Wird sie Einzug in die Standardsprache halten?}

Zunächst soll ein kurzer historischer Abriss zur Verwendung des Verbs stillen Anhaltspunkte für einen möglichen Bedeutungswandel geben:

Stillen als die natürliche Art, sein Baby zu ernähren, war schon immer ein nicht ganz konfliktfreies und problemloses Feld. In der Antike waren die Frauen den Männern untergeordnet, die Frau war zuständig für die Sicherung und „Aufzucht“ der Nachkommenschaft (vgl. Brodersen und Zimmermann 2006: 195). Besonders im Mittelalter wurde Maria häufig als Stillende inszeniert, die sog. Maria lactans (vgl. z. B. Schreiner 1994). Dass Herzeloyde ihren Parzival selbst stillt, ist eine extra Erwähnung wert (von Eschenbach 1998: 113, 5-16. B). Belege für einen möglichen Sprachwandel in den nachfolgenden Jahrhunderten bis in die Neuzeit bedürfen genauerer Textanalyse und stehen noch aus.

In der Zeit des Nationalsozialismus wird in Deutschland „Mutterschaft“ stark ideologisiert: Nur wer sein Kind stillt, erfüllt seine Pflicht als Mutter und kommt auch seiner „rassischen“ Verpflichtung nach (vgl. insbes. Haarer 1940: 116). Die Autorin empfiehlt einen festen Stillrhythmus von drei bzw. vier Stunden und die Einhaltung einer Nachtpause. Nur so werde das Kind Rhythmus und Disziplin lernen. Mindestens ein Jahr lang müsse das Kind gestillt werden. Nach dem Ende des Nationalsozialismus ist das Buch von Haarer unter dem Titel „Die Mutter und ihr erstes Kind“ bis 1986 in der damaligen Bundesrepublik Deutschland immer wieder aufgelegt und millionenfach verkauft worden. Selbstverständlich wurden die dezidiert nationalsozialistisch gefärbten Äußerungen getilgt, aber es , ,[...] blieb der Ratgeber nach wie vor geprägt von einem kühlen, distanzierten Umgang zwischen Mutter und Kind“" (Seichter 2014: 98). Der Diskurs ist politisch geprägt. Intendiert ist über einen längeren Zeitraum, dass solche „Vorschriften“ eingehalten werden sollen.

Im Jahr 1980 erschien erstmals „Das Stillbuch“ von Hannah Lohtrop, das lange Zeit als Standardwerk galt. Lohtrop hatte im Jahr 1977 in der BRD nach amerikanischem Vorbild die „La Leche Liga“ mitbegründet. Die Leche Liga legte den 
Grundstein für die „Stillbewegung“ in Deutschland. Erste Stillgruppen werden gegründet. Stillberaterinnen nehmen ihre Tätigkeit auf. Aus Sicht dieser Liga ist Stillen ein wichtiges Element für die gesunde Entwicklung eines Kindes, Muttermilch biete aus ernährungsphysiologischer Sicht einen optimalen Start ins Leben (vgl. www.lalecheliga.de). 1994 wurde am Robert-Koch-Institut die Nationale Stillkommission gegründet. Seit 2002 hat sie ihren Sitz beim Institut für Risikobewertung. Hauptaufgabe der Stillkommission ist die Förderung des Stillens in der BRD. Sie berät die Bundesregierung, gibt Richtlinien und Empfehlungen heraus und unterstützt Initiativen zur Beseitigung von Stillhindernissen (vgl. auch www.bfr.bund.de). Auf den Seiten der Kommission findet man auch die WHO-Empfehlung zum Stillen: Sechs Monate soll vollgestillt werden, die nationale Stillkommission empfiehlt, nicht vor dem 5. Lebensmonat und spätestens zu Beginn des 7. Monats mit Beikost zu beginnen. Sie betont, dass Mutter und Kind gemeinsam bestimmen, wann abgestillt wird. Aus den Jahren 1997/98 liegt die einzige bundesweite Studie zu Stillen und Säuglingsernährung vor, ebenfalls über die Seiten der Stillkommission abrufbar. Aus der Studie geht unter anderem hervor, dass 90 Prozent der Frauen nach der Geburt stillen, nach vier bis sechs Monaten noch 58 bzw. 48 Prozent. Die durchschnittliche Gesamtstilldauer betrug zu diesem Zeitpunkt 26 Wochen. Einzelne Studien aus Bayern und Berlin zeigen, dass es in den letzten 15 Jahren keine wesentlichen Veränderungen gegeben hat. Die Daten zeigen aber auch, dass die staatlichen Empfehlungen zur Stilldauer und die tatsächliche Stilldauer auseinandergehen.

In diesen etwa dreißig Jahren Diskussion zu diesem Thema vollzieht sich ein Wechsel von eher intuitivem Herangehen zu einem wissenschaftlich angeleiteten Handeln.

Ausgebildete Stillberaterinnen bemühen sich, in Einzelberatungen und in Stillgruppen, Frauen zu unterstützen. Im Internet finden Frauen Informationen und Unterstützung, wie etwa im Forum stillen-und-tragen.de. Dort kommen also immer mehr Frauen mit der intransitiven Verwendung des Verbs stillen in Berührung. Es handelt sich dabei um ein Aufeinandertreffen von Laien- und Experten-Kommunikation.

Spätestens jetzt, also zu Beginn der Jahrtausendwende, wird ein Wandel in den Auffassungen zu den Rollen von Mutter und Kind und zu ihrem aktiven bzw. passiven Verhalten während des Stillens artikuliert, das heißt auch sprachlich abgebildet.

Hier kann zunächst nur auf die Beispiele aus der Kommunikation der Mütter untereinander in dem genannten Internetforum eingegangen werden. Die Ableitung von Tendenzen im Sprachwandel muss umfangreicheren Untersuchungen vorbehalten bleiben.

\section{ZUR ENTWICKLUNG DER INTRANSITIVEN VERWENDUNG DES VERBS STILLEN}

Nach diesem kurzen historischen Abriss soll unter linguistischer und kulturwissenschaftlicher Perspektive die Entwicklung der intransitiven Verwendung des Verbs stillen diskutiert werden:

Wörter verändern ihre Bedeutungsextension, [...] wenn sich Verschiebungen in Beziehungsfeldern ergeben, wenn sich der Stellenwert eines Wortes im 
semantischen Gefüge des Wortschatzes verändert. Die Kommunizierenden, ihre Anschauungen, ihre sozialen Welten mit ihren Interessen unterliegen einem ständigen Wandel. Bedeutungswandel beginnt mit Veränderung des Sprachgebrauchs. [...] [Er] läßt sich nicht am isolierten Wort beobachten, sondern nur im Rede- und Textzusammenhang (Schippan 2002: 251).

Betrachten wir zwei Äußerungen zum Thema „erstes Stillen“ nach der Geburt:

\begin{abstract}
Nach einer Weile auf deinem Bauch beginnt dein Baby, sich zu entspannen und wohl zu fühlen. Bald siehst du es mit seinem kleinen Mund suchen, lutschen und schmatzen [...]. Neugeborene erschnuppern offenbar die Mutterbrust. [...] Wenn man sie lässt, krabbeln sie zum Erstaunen ihrer Mutter selbst zielsicher zur Nahrungsquelle hin. Dort angekommen, saugen sie sich mit weit offenem Mund an der Brust an [...]. (Lohtrop 2000: 133f.)

Einige Stunden nach der Geburt nehmen Sie das Kind zum ersten Mal an die Brust. [...] Die Hebamme pflegt die ersten Stillversuche zu überwachen [...]. Sie legen sich ein kleines Kopfkissen in die Ellenbeuge und legen darauf das Köpfchen des Kindes, und zwar ganz bequem. Das Köpfchen darf nicht nach hinten sinken, und der kleine Kopf soll gerade vor die Brustwarze zum Liegen kommen. Nun nehmen Sie die Brustwarze zwischen Zeigefinger und den dritten Finger der rechten Hand und schieben sie dem kleinen Kind rasch und mit einem Ruck tief in den Mund. (Haarer 1964: 120)
\end{abstract}

In der Darstellung bei Haarer wird das Neugeborene als hilfloses Wesen beschrieben, das mit viel Unterstützung, Hilfsmitteln und Hilfestellung an die Brust gebracht wird, um trinken zu können. Die Mutter soll die Brustwarze regelrecht in den Mund stopfen. Das Baby wird sprachlich als passives, hilfloses Wesen charakterisiert. Anders bei Lohtrop: Hier erscheint das Neugeborene als relativ selbstständiges, aktives Wesen. Es signalisiert sein Interesse zu saugen selbst, sucht und findet den Weg selbstständig und braucht keine Unterstützung beim richtigen Ansaugen.

Dem Wandel in den Ansichten zum Verhalten der Neugeborenen in den ersten Stunden nach der Geburt und in den folgenden Monaten bei der Aufnahme von Muttermilch, also beim Stillen, folgt auch ein Wandel im Sprachgebrauch des Verbs stillen. Linguistisch beschreibbar ist ein grammatischer Wandel vom transitiven Verb hin zur intransitiven Verwendung. Die oben genannten Beispiele belegen dies. Dieser Sprachwandel ist durch den Wandel semantischer Merkmale bedingt und folgt dem Prinzip der sprachlichen Innovation.

Sprache ist veränderbar, weil sie grundsätzlich auch innovativ benutzt werden kann. [...] Sprachkommunikation läuft nicht kausal ab, wie nach Naturgesetzen, sondern intentional, final, zweckgerichtet [...]; sie dient der Realisierung von Intentionen der Kommunikatoren (Sprecher/Verfasser), die meist auf die Beeinflussung des Verhaltens der Rezipienten (Hörer/Leser) gerichtet sind. (von Polenz 1991: 37f.) 
Aus dem zu stillenden, passiven Säugling wird das aktive Stillkind, welches seine Bedürfnisse kennt und deren Befriedigung nicht nur einfordert, sondern auch aktiv vollzieht, sozusagen selbst in die Hand nimmt. In den oben genannten Beispielen scheint sich der intransitive Gebrauch des Verbs stillen durchzusetzen, auch weil offensichtlich ein „bewusster Bedeutungswandel“ (vgl. Fritz 1984: 750) stattfindet.

In der deutschen Sprache fehlt ein eigenständiges Wort für das, was das Kind da gerade aktiv tut, wenn es ohne fremde Hilfe allein den Weg an die Brust findet und daran saugt. Die Mutter ist auf das aktive Saugen ihres Kindes angewiesen, damit sich Milch bilden kann. Das Verb trinken ist zu unspezifisch und wird eher mit Flaschennahrung (bzw. generell Flüssigkeit) zu sich nehmen assoziiert. Das Verb saugen ist auch eher unpassend, da nicht alle Bedeutungsmerkmale des gemeinten Denotats abgebildet werden. Das Kind „saugt" nicht nur, sondern „,melkt" und „schluckt" auch. Außerdem könnte eine aktive Verwendung des Verbs „,saugen“, z. B. das Baby saugt, für Irritation sorgen, weil als erstes die hauswirtschaftliche Tätigkeit assoziiert werden könnte. Da das, was das Kind tut, eigentlich „melken“ meint bzw. ist (i.e.S. des Denotats), dieses Wort aber für die Gewinnung der Milch von Tieren verwendet wird, also ein anderes Denotat im Sprachgebrauch abbildet, ist die Verwendung des Verbs „melken“ nicht adäquat.

Die Sätze das Baby trinkt, das Baby saugt, das Baby melkt bilden zwar sprachlich die aktive Rolle des Kindes bei der Aufnahme von Muttermilch ab, setzen sich aber, zumindest in dem genannten Internetforum, in der Kommunikation zurzeit nicht durch. Um eine vermeintliche lexikalische Lücke zu füllen, finden sich seit 2015 häufiger Belege für eine Zunahme der intransitiven Verwendung des Verbs stillen, die es sogar möglich erscheinen lässt, dass der transitive Gebrauch des Verbs in absehbarer Zeit stark zurückgedrängt und nicht mehr als standardsprachliche Variante genutzt wird. Für die Ableitung solch einer Entwicklung ist allerdings eine umfangreiche Analyse notwendig.

Neben dieser kurzen Beschreibung eines grammatischen Wandels des Verbs stillen, der auf Bedeutungs- und kommunikativen Wandel fußt, sind neue Wortbildungen zu beobachten. In der Regel werden diese neuen Verben aber transitiv verwendet:

Welche Milchpumpe könnt ihr empfehlen? Ich pumpstille nun schon seit August. [...] Wie stelle ich das denn am besten an, rein technisch? Ich habe hier auch schon oft von Regeln gelesen, die Ihr beim tandemstillen für die großen Kinder habt. [...] Vielleicht werde ich sie nachts im nächsten halben Jahr abstillen. Es sei denn meine Kleine belehrt mich wieder eines Besseren und stillt sich von allein ab oder ich möchte nachts absolut nicht mehr stillen. (www.stillen-und-tragen.de, 26.03.2018)

Insgesamt kann eingeschätzt werden, dass sich allmählich ein Wandel vom transitiven Gebrauch des Verbs stillen zur intransitiven Verwendung vollzieht. Allerdings kann hier nicht vorhergesagt werden, ob der veränderte Blick auf das Kind in seiner aktiven Rolle beim Stillen tatsächlich zur Durchsetzung der intransitiven Variante des Verbs in der standardsprachlichen Kommunikation führen wird [eben, s. o.]. Noch beschränkt sich der intransitive Gebrauch eher auf bestimmte Internetplattformen. In anderen Kommunikationssituationen, vor allem auch wenn verschiedene 
Elterngenerationen aufeinander treffen, stößt die intransitive Verwendung des Verbs auf Widerstand und Irritation. Vermutlich werden transitive und intransitive Verwendung des Verbs stillen eine längere Zeit nebeneinander existieren. Dieses Beispiel zeigt eindrucksvoll den Beginn einer eher evolutionären semantischen Veränderung in der Lexik der Gegenwartssprache.

\section{Literatur}

BRODERSEN, Kai/Bernhard, ZIMMERMANN (2006) Metzler Lexikon Antike. Stuttgart/ Weimar: Metzler.

ESCHENBACH, Wolfram von (1998) Parzival. Berlin/New York: Walter de Gruyter. FRITZ, Gerd (1984) „Ansätze zur Theorie des Bedeutungswandels.“ In: W. Besch et al. (Hrsg.), Sprachgeschichte I. Berlin/New York: de Gruyter, 739-753.

HAARER, Johanna (1940) Die deutsche Mutter und ihr erstes Kind. München: Lehmann.

HAARER, Johanna (1964) Die Mutter und ihr erstes Kind. München: Lehmann.

HEUSINGER, Siegfried (2004) Die Lexik der deutschen Gegenwartssprache. Eine Einführung. München: Fink.

KÖBLER, Gerhard (1994) Taschenwörterbuch des althochdeutschen Sprachschatzes. Paderborn: UTB.

KLUGE, Friedrich (2002) Etymologisches Wörterbuch der deutschen Sprache. Berlin/ New York: de Gruyter.

LEXER, Matthias (1992) Mittelhochdeutsches Taschenwörterbuch. Mit Nachträgen von Ulrich Pretzel. Stuttgart: Hirzel.

LOHTROP, Hannah (2000) Das Stillbuch. München: Kösel.

POLENZ, Peter von (1991) Deutsche Sprachgeschichte vom Spätmittelalter bis zur Gegenwart. Band I. Berlin/New York: de Gruyter.

SCHIPPAN, Thea (2002) Lexikologie der deutschen Gegenwartssprache. Tübingen: Niemeyer.

SCHREINER, Klaus (1994) Maria. Jungfrau, Mutter, Herrscherin. München [etc.]: Hanser.

SEICHTER, Sabine (2014) Erziehung an der Mutterbrust. Eine kritische Kulturgeschichte des Stillens. Weinheim/Basel: Beltz Juventa.

WAHRIG, Gerhard (1982) Der kleine Wahrig. Wörterbuch der deutschen Sprache. München: dtv.

\section{Internetquellen}

Forum Stillen und Tragen. www.stillen-und-tragen.de [03.01.2019].

La Leche Liga. www.lalecheliga.de/la-leche-liga/wer-wir-sind/unsere-arbeit [29.03.2018]. Nationale Stillkommission.www.bfr.bund.de/de/nationale_stillkommission [27.03.2018]. 


\section{Zusammenfassung \\ LEXIKALISCHER WANDEL AM BEISPIEL DES VERBS STILLEN}

Sprachwandel in der Grammatik vollzieht sich relativ langsam. In den meisten Fällen ist grammatischer Wandel mit Veränderungen in der Semantik der Lexik verbunden. Beide Veränderungsprozesse können sich wechselseitig bedingen. So kann z. B. Bedeutungswandel im Bereich der Verben Valenzänderungen hervorrufen, die die Anzahl und Semantik der Ergänzungen im Satz beeinflussen können. In diesen Prozess sind Veränderungen hinsichtlich Transitivität und Intransitivität einzelner Verben eingebettet.

Im Beitrag wird nur ein Beispiel für lexikalischen Wandel kurz beschrieben, ohne zum jetzigen Zeitpunkt eine valide empirische Untersuchung vorzulegen. Mögliche dialektale Varianten in der Verwendung des Verbs bleiben unberücksichtigt.

Das bisher transitiv verwendete Verb stillen wird in bestimmten Medien wie zum Beispiel in einem Internetforum zunehmend intransitiv gebraucht. Dieser Sprachwandel steht im Zusammenhang mit dem veränderten Blick auf physische und psychische Fähigkeiten von Säuglingen, denen eine aktivere Rolle beim Stillen zugeschrieben wird. Diese Rollenänderung gehe mit einem Wandel des Menschenbildes in der Gesellschaft und Kultur einher, so erste Überlegungen von Psychologen. Beispiele aus einem Internetforum belegen einen zunehmenden intransitiven Gebrauch des Verbs stillen und somit die sprachliche Innovation in der Lexik der deutschen Gegenwartssprache.

Schlüsselwörter: stillen, Sprachwandel, Internetforum, Bedeutungswandel, Transitivität

\section{Abstract \\ LEXICAL CHANGE ON THE THE EXAMPLE OF THE VERB STILLEN}

Language change in grammar is relatively slow. In most cases, grammatical change is associated with changes in lexical semantics. Both types of change can be interrelated. For example, a change in verb meaning can cause valence changes which can influence the number and semantics of the complements in the sentence. Part of this process are also changes concerning transitivity and intransitivity of individual verbs.

The article briefly describes only one example of lexical change without presenting a valid empirical study at this point. Possible dialectal variants in the use of the verb are not considered.

The previously transitive German verb stillen (Engl. 'breastfeed') is used increasingly intransitively in certain media, such as internet forums. This instance of language change is related to the changed view of the physical and psychological abilities of infants, to whom a more active role in breastfeeding is attributed. According to initial considerations by psychologists, role changes go hand in hand with changes in the way the human being is perceived in social and cultural terms. Examples from an internet 
forum show an increasing intransitive use of the verb stillen as an innovation in the lexicon of contemporary German.

Keywords: stillen, language change, internet forum, meaning change, transitivity

Povzetek

\section{LEKSIKALNA SPREMEMBA NA PRIMERU GLAGOLA STILLEN}

Jezik se na področju slovnice spreminja relativno počasi. V večini primerov so spremembe v slovnici vezane na spremembe leksikalne semantike. Ob vrsti procesov spreminjanja pa se lahko obe vrsti sprememb obojestransko pogojujeta. Tako lahko na primer sprememba pomena pri glagolih izzove spremembe vezljivosti, kar vpliva na število in pomen določil $\mathrm{v}$ stavku. $\mathrm{V}$ te procese so vključene tudi spremembe glagolske prehodnosti in neprehodnosti.

$\mathrm{V}$ prispevku je opisan le en primer leksikalne spremembe, ki se v sedanjem trenutku ne opira na empirično raziskavo. Ev. narečne različice rabe glagola niso bile upoštevane.

Do sedaj tranzitivno rabljeni glagol stillen (slow. 'dojiti') se v določenih medijih kot na primer $\mathrm{v}$ na spletnih forumih vse pogosteje uporablja intranzitivno. Ta jezikovna sprememba je povezana s spremenjenim pogledom na fizične in psihične sposobnosti dojenčkov, ki jim je pri dojenju pripisana aktivnejša vloga. Ta sprememba vlog naj bi sovpadala s spremenjeno predstavo o človeku v družbi in kulturi, kot so to začeli razlagati psihologi. Primeri s spletnega foruma dokazujejo naraščanje intranzitivne rabe glagola stillen in s tem jezikovne prenovitve v besedju sodobnega nemškega jezika.

Ključne besede: stillen, jezikovno spreminjanje, spletni forum, pomenska sprememba, prehodnost 